\title{
Prevalência da hipertensão arterial sistêmica em crianças da rede pública estadual de ensino de Carmo do Cajuru-MG
}

RESUMO | Objetivo: Analisar estudantes dos sextos anos das escolas públicas estaduais do município de Carmo do Cajuru/MG com o intuito de identificar aqueles com predisposição e prevalência à Hipertensão Arterial Sistêmica. Método: Pesquisa quantitativa, descritiva, de delineamento transversal. Foi utilizado questionário estruturado destinados aos pais ou responsáveis legais pelas crianças, bem como a realização de medidas antropométricas e aferição da pressão arterial. As variáveis de interesse foram analisadas e apresentadas utilizando a estatística descritiva, média, frequência absoluta e porcentagem. Resultados: A amostra estudada contou com a participação de 119 (64 meninas e 53 meninos) das quais 65,5\% com 11 anos e 33,9\% com 12 anos. Com relação à média da pressão arterial 100\% dos meninos e 93,7\% das meninas dispuseram dentro dos limites normais. Conclusão: A partir da realização deste estudo, vemos que se fazem necessárias reflexões sobre agentes considerados como fatores de risco para o desenvolvimento da hipertensão.

Palavras-chaves: Criança; Estudante; Pressão Arterial; Saúde Pública.

\begin{abstract}
Objective: To analyze sixth year students from state public schools in the municipality of Carmo do Cajuru / MG in order to identify those with a predisposition and prevalence to Systemic Arterial Hypertension. Method: Quantitative, descriptive, cross-sectional research. A structured questionnaire was used for parents or legal guardians of the children, as well as anthropometric measurements and blood pressure measurements. The variables of interest were analyzed and presented using descriptive statistics, mean, absolute frequency and percentage. Results: The studied sample counted with the participation of 119 (64 girls and 53 boys) of which $65.5 \%$ were 11 years old and 33.9\% were 12 years old. Regarding the mean blood pressure, $100 \%$ of the boys and $93.7 \%$ of the girls were within normal limits. Conclusion: From the realization of this study, we see that it is necessary to reflect on agents considered to be risk factors for the development of hypertension.
\end{abstract}

Keywords: Child; Student; Blood pressure; Public health.

RESUMEN | Objetivo: Analizar estudiantes de sexto año de escuelas públicas del municipio de Carmo do Cajuru / MG para identificar a aquellos con predisposición y prevalencia de hipertensión arterial sistémica. Método: Investigación cuantitativa, descriptiva, transversal. Se utilizó un cuestionario estructurado para los padres o tutores legales de los niños, así como mediciones antropométricas y mediciones de la presión arterial. Las variables de interés se analizaron y presentaron mediante estadística descriptiva, media, frecuencia absoluta y porcentaje. Resultados: La muestra estudiada contó con la participación de 119 (64 niñas y 53 niños) de los cuales 65.5\% tenían 11 años y 33.9\% tenían 12 años. En cuanto a la presión arterial media, el $100 \%$ de los niños y el $93,7 \%$ de las niñas se encontraban dentro de los límites normales. Conclusión: a partir de la realización de este estudio, vemos que es necesario reflexionar sobre los agentes considerados factores de riesgo para el desarrollo de hipertensión. Palavras claves: Niño; Estudiante; Presión arterial; Salud pública.

\section{Valéria Aparecida da Silva}

Enfermeira. Graduação em Enfermagem pela Universidade do Estado de Minas Gerais (UEMG), Unidade Divinópolis.

ORCID: 0000-0003-0095-5520

\section{Tatiane Nogueira Rabelo}

Enfermeira. Graduação em Enfermagem pela Universidade do Estado de Minas Gerais (UEMG), Unidade Divinópolis. ORCID: 0000-0003-4138-0874

\section{Fernanda Marcelino de Rezende e Silva}

Mestre em Enfermagem pela UFMG, Docente do Curso de Graduação em Enfermagem da Universidade do Estado de Minas Gerais (UEMG), Unidade Divinópolis. ORCID: 0000-0003-2236-7009

Recebido em: 17/07/2020

Aprovado em: 28/07/2020
Karla Amaral Nogueira Quadros

Enfermeira. Mestre em Educação, Cultura e Organização Social pela Universidade do Estado de Minas Gerais. Minas Gerais, Brasil. Docente do Curso de Graduação em Enfermagem da Universidade do Estado de Minas Gerais (UEMG), Unidade Divinópolis. ORCID: 0000-0002-3750-4873

\section{Regina Consolação dos Santos}

Mestre em Ciências pela Universidade Federal de São João Del Rei, (UFSJ). Docente do Curso de Graduação em Enfermagem da Universidade do Estado de Minas Gerais (UEMG), Unidade Divinópolis.

ORCID: 0000-0002-7393-3210

\section{Silmara Nunes Andrade}

Enfermeira. Doutora em Ciências da Saúde pela Universidade Federal de São João Del Rei (UFSJ). Docente do Curso de Graduação em Enfermagem da Universidade do Estado de Minas Gerais (UEMG), Unidade Divinópolis. ORCID: 0000-0002-1975-0827
INTRODUÇÃO

$\Lambda$ $\mathrm{s}$ doenças cardiovasculares (DCV) atualmente se destacam de maneira muito evidente no que tange a causa de mortes prematuras. A hipertensão arterial sistêmica (HAS) é considerada como o principal fator de risco para o desenvolvimento das DCV, constituindo um problema de saúde pública em âmbito mundial ${ }^{(1,2,3)}$.

A hipertensão é uma condição na qual a força que o sangue faz contra as paredes das artérias é muito grande de acordo com a idade do indivíduo. Está comumente associada com a idade adulta, entretanto tal manifestação têm raízes na infância, por se tratar de uma patologia de acometimento silencioso e assintomático. A literatura trás que crianças 
com níveis elevados da pressão arterial apresentam maior probabilidade de se tornarem adultos hipertensos ${ }^{(2,4)}$.

Nas últimas décadas, a prevalência de Pressão Arterial (PA) elevada tem acrescido entre as crianças, e a carência de diagnóstico pode levar ao não tratamento da enfermidade e sua persistência até a idade adulta $^{5}$. Estudos nacionais a prevalência de PA alterada em crianças varia entre 3,6 e $15,8 \%^{2}$. Desta forma, a literatura traz que é indispensável à medida da PA a partir dos três anos de idade, uma vez por ano, ou antes, disso, quando a criança possuir fatores de risco ${ }^{(2,4)}$.

$\mathrm{O}$ alto número de acometimentos de HAS na infância está adjunto aos fatores de risco nos quais estas crianças são expostas. Precisamente a fatores genéticos, ao excesso de peso, baixo peso ao nascer e ausência de Aleitamento Materno (AM) influenciam no desenvolvimento de níveis pressóricos elevados. O estilo de vida que as faixas mais jovens seguem são também agregados com a elevação da PA, como os maus costumes alimentares e hábitos de vida sedentários ${ }^{(5)}$.

Apesar de, na maioria dos casos, não possuir cura para a HAS, sabe-se que há tratamentos para controlá-la. Os tratamentos não medicamentosos devem ser aderidos pelos indivíduos que estão com alterações na pressão arterial sistêmica e os demais indivíduos que tenham disposições a se tornarem hipertensos. As medidas incluem a praticar atividade física regularmente, ter hábitos alimentares balanceados. E o tratamento medicamentoso é dado por um médico feito a partir da administração de medicamentos mais indicado para o caso do individuo ${ }^{(3)}$.

Considerando que pesquisas, têm mostrado um crescimento de acometimentos de hipertensão na infância devido à exposição cada vez mais precoce aos fatores de risco, torna-se necessário a avaliação do perfil desses indivíduos, pois é de fundamental relevância para o município e equipes de saúde da localidade saberem e caso necessário realizarem ações voltadas para esse âmbito ${ }^{(2,4)}$.
Por intermédio da detecção precoce da HAS em crianças torna-se possível o controle e menor probabilidade de desencadeamento e/ou agravamento da hipertensão. Desta forma, o estudo teve com objetivo de analisar estudantes dos sextos anos das escolas públicas estaduais do município de Carmo do Cajuru-MG com o intuito de identificar aqueles com predisposição e prevalência à Hipertensão Arterial Sistêmica.

\section{MÉTODO}

Trata-se de um estudo descritivo, transversal de abordagem quantitativa, realizado em seis escolas públicas do município de Carmo do Cajuru-MG nas quais aceitaram participar do estudo.

Trata-se de um estudo descritivo, transversal de abordagem quantitativa, realizado na cidade de Divinópolis, Minas Gerais. Carmo do Cajuru é um município de 22.478 habitantes 6, localizado no Centro-Oeste do estado de Minas Gerais.

Segundo dados obtidos na Secretaria Estadual da Educação de Minas Gerais/Subsecretária de Desenvolvimento da Educação Básica (2018), o mesmo conta com seis escolas públicas estaduais, distribuídas em urbanas e rurais nas quais têm matriculados cerca de 202 alunos cursando o sexto ano em 2018. Assim, para participar do estudo foram selecionadas crianças de até 12 anos 11 meses e 29 dias, nas quais cursam os $6^{\circ}$ anos de tais escolas.

A coleta de dados foi feita no segundo semestre de 2018, por meio de questionários estruturados, destinados aos pais ou responsáveis legais pelas crianças a responderem, bem como a realização de medidas antropométricas (mensuração de peso e altura) e aferição da pressão arterial, pelas pesquisadoras nas crianças participantes do estudo. Ressalta-se que os alunos participantes da pesquisa receberam o Termo de Assentimento e o responsável legal o Termo de Consentimento Livre e Esclarecido, por 
meio do qual foram informados sobre a realização e os objetivos do estudo. Somente foram analisadas as crianças cujos responsáveis legais concordaram com a realização do estudo.

Entre as variáveis sociodemográficas, foi investigado: sexo, idade, cor/raça, renda familiar e escolaridade dos pais ou responsáveis legal. A idade foi apresentada em anos completos. A cor/raça foi indagada se branca, parda ou negra. A classe econômica foi avaliada de acordo com os salários mínimos recebidos pela família: até um salário mínimo, até dois e até três ou mais. A escolaridade foi ponderada em ensino fundamental, médio e superior, sendo os mesmos completos e/ ou incompletos.

Dentre as variáveis de saúde, pesquisou-se o peso ao nascer, idade gestacional, tempo de amamentação exclusiva no peito e histórico Hipertensão Arterial Sistêmica e Diabetes Mellitus (DM). O peso ao nascer foi classificado em: < $2.500 \mathrm{~kg}$ (baixo peso), $2.500 \mathrm{~kg}$ a $2.999 \mathrm{~kg}$ (peso insuficiente), $3.000 \mathrm{~kg}$ a $3.999 \mathrm{~kg}$ (peso adequado) e $>4.000 \mathrm{~kg}$ (macrossômico). O tempo de gestação indagará se prematuro $(<37$ semanas) ou não prematuro ( $>37$ semanas). O tempo de amamentação investigou se o aleitamento materno foi inferior a seis meses, igual ou superior a seis meses ou não amamentou. Quanto às doenças relatadas pelos indivíduos, foram consideradas tanto as pessoais quanto as familiares (pais, avós e outros). A anamnese continha algumas doenças pré-estabelecidas como: diabetes mellitus e HAS.

Para variáveis de estilo de vida considerou hábitos alimentares, práticas de atividades físicas, e horas de lazer tecnológico. Nos hábitos alimentares averiguou a frequência de consumo de frutas e fast-foods, em nenhuma, uma, duas e três ou mais. A prática de atividades físicas apurou se pratica e não pratica quais e quantas vezes por semana. As horas de lazer tecnológico foram delimitadas em até uma hora, até duas horas e três horas ou mais.
Foram realizadas medidas de peso, estatura, IMC e pressão arterial. A pesagem foi realizada com crianças descalças, vestindo uniforme escolar, em uma balança digital. A estatura procedeu com crianças descalças, vestindo uniforme escolar, em posição ereta, encostados numa superfície plana vertical, braços pendentes com as mãos espalmadas sobre as coxas, os calcanhares unidos e as pontas dos pés afastadas, formando um ângulo de $60^{\circ}$, joelhos em contato e cabeça erguida. Utilizou fita métrica de dois metros. O IMC foi calculado por meio da divisão do peso corporal pela estatura ao quadrado e classificado de acordo com a idade na Norma Técnica do Sistema de Vigilância Alimentar e Nutricional7, sendo magreza acentuada ( $<$ percentil 0,1$)$, magreza $(\geq$ percentil 0,1 e < percentil 3), eutrofia (> percentil 3 e $<$ percentil 85), sobrepeso (> percentil 85 e $\leq$ percentil 97), obesidade (>percentil 97 e $\leq$ percentil 99,9) e obesidade grave (> percentil 99,9).

A mensuração da PA foi realizada no ambiente escolar, com explicação e demonstração dos procedimentos para mensuração de acordo com os parâmetros da idade citados na $7^{a}$ Diretriz brasileira de Hipertensão Arterial ${ }^{3}$. Tais procedimentos incluíram a condição inicial de repouso, com o indivíduo sentado, o braço esquerdo na posição supina e apoiado na altura do coração. O equipamento utilizado para aferição foi um aparelho de pressão previamente calibrado com manguitos proporcionais à circunferência do braço da criança. Foram mensuradas a pressão arterial sistólica (PAS) e diastólica (PAD), sendo as medidas aferidas durante os períodos escolares: matutino (7:00 às 11:30 h) e vespertino (13:00 às 17:30 h). $\mathrm{O}$ agendamento foi realizado de acordo com a disponibilidade de horário que cada escola ofertou, sendo antes ou depois do intervalo e/ou até mesmo nas aulas de Educação Física. Deste modo, foram considerados 5 minutos de descanso sentado do avaliado em condições de repouso, e mínimo de 10 a 15 minutos caso estiver realizando atividades físicas 
Tabela 1 - Características sociodemográficas dos alunos participantes e seus pais ou responsáveis legais, 2018, Carmo do Cajuru (MG).

\begin{tabular}{|c|c|c|}
\hline Variáveis & $\mathrm{N}$ & $\%$ \\
\hline Idade & $n(118)$ & \\
\hline 11 & 78 & 66,1 \\
\hline 12 & 40 & 33,9 \\
\hline Total & 118 & 100,0 \\
\hline Sexo & $\mathrm{n}(117)$ & \\
\hline Feminino & 64 & 54,7 \\
\hline Masculino & 53 & 45,3 \\
\hline Total & 117 & 100,0 \\
\hline Raça & n (118) & \\
\hline Branca & 52 & 44,0 \\
\hline Negra & 10 & 8,5 \\
\hline Parda & 56 & 47,5 \\
\hline Total & 118 & 100,0 \\
\hline Escolas & n (119) & \\
\hline Rurais & 27 & 22,7 \\
\hline Urbanas & 92 & 77,3 \\
\hline Total & 119 & 100 \\
\hline Renda familiar & n (114) & \\
\hline Até um salário mínimo & 44 & 38,6 \\
\hline Até dois salários mínimos & 48 & 42,1 \\
\hline Três salários mínimos ou mais & 22 & 19,3 \\
\hline Total & 114 & 100,0 \\
\hline Tipo de moradia & n (111) & \\
\hline Alugada & 26 & 23,4 \\
\hline Própria & 85 & 76,6 \\
\hline Total & 111 & 100,0 \\
\hline Escolaridade da mãe e/ou da responsável legal pela criança & n (117) & \\
\hline Ensino Fundamental Completo & 11 & 9,4 \\
\hline Ensino Fundamental Incompleto & 35 & 29,9 \\
\hline Ensino Médio Completo & 37 & 31,6 \\
\hline Ensino Médio Incompleto & 18 & 15,4 \\
\hline Ensino Superior Completo & 7 & 6,0 \\
\hline Ensino Superior Incompleto & 9 & 7,7 \\
\hline Escolaridade do pai e/ou responsável legal pela criança & n (112) & \\
\hline Ensino Fundamental Completo & 6 & 5,3 \\
\hline Ensino Fundamental Incompleto & 50 & 44,7 \\
\hline Ensino Médio Completo & 28 & 25,0 \\
\hline Ensino Médio Incompleto & 19 & 17,0 \\
\hline Ensino Superior Completo & 3 & 2,7 \\
\hline
\end{tabular}

e a próxima aferição será de no mínimo dois minutos depois da primeira.

A classificação ocorreu de acordo com a idade pela $7^{a}$ Diretriz brasileira de Hipertensão Arterial da Sociedade brasileira de Cardiologia (2016) em normotensos (PA < percentil 90), limítrofe (PA entre percentis 90 a 95), hipertensão estágio 1 (percentil 90 a 95 mais $5 \mathrm{mmHg}$ ) e hipertensão estágio 2 (PA > percentil 99 mais $5 \mathrm{mmHg}$ ).

Para a apresentação das variáveis do estudo, utilizou-se a estatística descritiva, média, frequência absoluta e porcentagem. Este estudo foi realizado de acordo com os princípios que orientam a realização de pesquisa envolvendo seres humanos, conforme a resolução 466/2012, e todos os procedimentos que envolveram os sujeitos foram aprovados pelo Comitê de Ética em Pesquisa em Seres Humanos da Fundação Educacional de Divinópolis - FUNEDI parecer $\mathrm{n}^{\circ}$ 2.928.131, CAAE $\mathrm{n}^{\circ}$ 95890718.4.0000.5115.

\section{RESULTADOS}

A amostra estudada contou com a participação de 119 alunos, constituindo estes majoritariamente a idade de 11 anos os quais correspondem $66,1 \%$ da amostra sendo 78 alunos, seguido por 40 estudantes com 12 anos representando $33,9 \%$. Nota-se a proporção de 64 estudantes $(54,7 \%)$ do sexo feminino e os demais 53 alunos $(45,3 \%)$ do sexo masculino. A raça com maior evidência foi à parda com um total de 56 indivíduos $(47,5 \%)$ seguida da progênie branca com $44 \%$ no qual contabiliza um total de 52 alunos. Observa-se que $22,7 \%$ das crianças em estudo são estudantes das escolas rurais, o que contabiliza um total de 27 alunos e os demais 92 educandos $(77,3 \%)$ são provenientes das instituições urbanas, conforme (TABELA 1).

Em continuidade (TABELA 1), pertinente à renda familiar percebe-se o destaque de 48 das famílias cujo recebem até dois salários mínimos representando $40,3 \%$ do estudo, procedendo aquelas 
Ensino Superior Incompleto

Total

Fonte: as autoras (2018)

Tabela 2 - Dados gestacionais e primeiros meses de vida dos alunos participantes, 2018, Carmo do Cajuru (MG).

\begin{tabular}{lcc} 
Variáveis & $\mathbf{N}$ & $\%$ \\
\hline Peso ao nascer & $\mathbf{n}(119)$ & \\
\hline Menor que $2.500 \mathrm{~kg}$ & 16 & 13,4 \\
\hline Entre $2.501 \mathrm{~kg}$ e $2.999 \mathrm{~kg}$ & 32 & 26,9 \\
\hline Entre $3.000 \mathrm{~kg}$ e $3.999 \mathrm{~kg}$ & 65 & 54,7 \\
\hline Maior que $4.000 \mathrm{~kg}$ & 6 & 5,0 \\
\hline Total & 119 & 100,0 \\
\hline Idade gestacional do nascimento & $\mathbf{n}(118)$ & \\
\hline Maior que 37 semanas & 86 & 72,9 \\
\hline Menor que 37 semanas & 32 & 27,1 \\
\hline Total & 118 & 100,0 \\
\hline Tempo de amamentação do leite materno & $\mathbf{n}(118)$ & \\
\hline Igual ou superior a seis meses & 70 & 59,3 \\
\hline Inferior a seis meses & 41 & 34,7 \\
\hline Não amamentou & 7 & 6,0 \\
\hline Total & 118 & 100,0 \\
\hline Mãe na gravidez teve diabetes gestacional & $\mathbf{n}(119)$ & \\
\hline Não & 117 & 98,3 \\
\hline Sim & 2 & 1,7 \\
\hline Total & 119 & 100,0 \\
\hline
\end{tabular}

Fonte: as autoras (2018)

Tabela 3 - Presença de DCNT nos familiares e nos alunos participantes, 2018, Carmo do Cajuru (MG).

\begin{tabular}{lcc} 
Variáveis & $\mathbf{N}$ & $\%$ \\
\hline Tem hipertensão & $\mathbf{n}(\mathbf{1 1 6})$ & \\
\hline Não & 94 & 81,0 \\
\hline Não sabe & 17 & 14,7 \\
\hline Sim & 5 & 4,3 \\
\hline Total & 116 & 100,0 \\
\hline Histórico de hipertensão na família & $\mathbf{n}(119)$ & \\
\hline Não & 21 & 17,6 \\
\hline Sim & 98 & 82,4 \\
\hline Total & 119 & 100,0 \\
\hline Histórico de diabetes mellitus na família & $\mathbf{n ( 1 1 4 )}$ & \\
\hline Não & 51 & 44,7 \\
\hline Sim & 63 & 55,3
\end{tabular}

nas quais recebem até um salário mínimo contando com um total de 44 indivíduos $(37 \%)$. Vê-se que a maioria dos estudantes $(71,0 \%)$ moram em residência própria sendo 85 alunos da amostra. A escolaridade das mães ou responsáveis legais pelas crianças se baseia em ensino médio completo 37 (31,1\%) seguido pelo ensino fundamental completo 35 (29,4\%). O ensino fundamental incompleto é a escolaridade da maioria dos pais ou responsáveis legais pelas crianças contando com um total de 50 homens representando $42 \%$ da amostra.

Os dados gestacionais e dos primeiros meses de vida são mostrados na (TABELA 2). Nota-se a maioria das crianças $(54,7 \%)$ com um peso de $3.000 \mathrm{Kg}$ e $3.999 \mathrm{Kg}$ ao nascer correspondendo a 65 alunos. 72,9\% dos alunos (86) estudados nasceram com a idade gestacional maior que 37 semanas. O tempo de amamentação teve o destaque com 70 crianças cuja porcentagem representou 59,3\%, sendo igual ou superior a seis meses. Em relação ao diabetes gestacional, da amostra obtida, em 98,3\% (117) alunos a mãe não teve diabetes durante o período gestacional.

Relacionado às Doenças Crônicas não Transmissíveis (DCNT), como apontadas na (TABELA 3) percebe-se que a maioria dos alunos em estudo (81\%) não apresentam hipertensão sendo um total de 94 estudantes. Pertinente à família, vê-se que 98 alunos (82,4\%) têm casos de hipertensão. Dentre os familiares com maior número de citações foram avó materna, avó paterna, avô materno, avô paterno, outros parentes, mãe e pai, respectivamente. Relacionado ao Diabetes Mellitus 55,3\% (63) dos estudantes referiram familiares com tal patologia. Neste os com maiores números de menções foram avó materna, outros parentes, avó paterna, avô paterno e materno, mãe e pai, concomitantemente.

Em relação ao estilo de vida das crianças, conforme descritos na (TABELA 04), observa-se a ocorrência de 44 alunos consumindo apenas uma fruta ao 


\begin{tabular}{|c|c|c|}
\hline Total & 114 & 100,0 \\
\hline \multicolumn{3}{|l|}{ Fonte: as autoras (2018) } \\
\hline \multicolumn{3}{|c|}{ Tabela 4 - Estilo de vida dos alunos participantes, 2018, Carmo do Cajuru (MG). } \\
\hline Variáveis & $\mathbf{N}$ & $\%$ \\
\hline Consume quantas frutas por dia & n (117) & \\
\hline Nenhuma & 22 & 18,8 \\
\hline Uma & 44 & 37,6 \\
\hline Duas & 25 & 21,4 \\
\hline Três ou mais & 26 & 22,2 \\
\hline Total & 117 & 100,0 \\
\hline Consome quantos fast-foods por dia & n (117) & \\
\hline Nenhum & 31 & 26,5 \\
\hline Um & 38 & 32,5 \\
\hline Dois & 26 & 22,2 \\
\hline Três ou mais & 22 & 18,8 \\
\hline Total & 117 & 100,0 \\
\hline Horas de lazer tecnológico por dia & n (117) & \\
\hline Até uma hora & 28 & 23,9 \\
\hline Até duas horas & 44 & 37,6 \\
\hline Três horas ou mais & 45 & 38,5 \\
\hline Total & 117 & 100,0 \\
\hline Realiza atividade física & n (119) & \\
\hline $\operatorname{Sim}$ & 84 & 75,1 \\
\hline Não & 35 & 29,4 \\
\hline Total & 119 & 100,0 \\
\hline Quantas vezes realiza atividade física por semana & $n(82)$ & \\
\hline $1 \mathrm{dia}$ & 8 & 9,7 \\
\hline 2 dias & 40 & 48,8 \\
\hline 3 dias & 15 & 18,3 \\
\hline 4 dias & 7 & 8,5 \\
\hline 5 dias ou mais & 12 & 14,7 \\
\hline Total & 82 & 100,0 \\
\hline
\end{tabular}

Fonte: as autoras (2018)

\section{Tabela 5 - IMC e Média da PA dos alunos participantes, 2018, Carmo do Cajuru (MG).}

\begin{tabular}{lcccc} 
Variáveis & \multicolumn{3}{c}{ Meninas } & \multicolumn{2}{c}{ Meninos } \\
& N & $\%$ & $\mathbf{N}$ & $\%$ \\
IMC & $\mathbf{n}(64)$ & & $\mathbf{n}(55)$ & \\
Magreza Acentuada $(<$ Percentil 0,1) & - & - & - & - \\
\hline Magreza ( $\geq$ Percentil 0,1 e < Percentil 3) & 4 & 6,3 & 1 & 1,8 \\
\hline Eutrofia (> Percentil 3 e < Percentil 85) & 47 & 73,4 & 43 & 78,2 \\
Sobrepeso (> Percentil 85 e $\leq$ Percentil 85) & 11 & 17,2 & 7 & 12,7
\end{tabular}

dia $(37,6 \%) .32,5 \%$ dos indivíduos ingerem ao dia um tipo de fast-foods, equivalendo a 38 estudantes. Pertinente ao lazer tecnológico $38,5 \%$ (45) da amostra fazem três horas ou mais, seguido por aqueles de até duas horas com $37,6 \%$ (44) do estudo. Relacionado à atividade física a maioria dizem realizar sendo contabilizados 84 alunos correspondendo a $75,1 \%$. Dentre as atividades mais desenvolvidas citam futebol, caminhada e andar de bicicleta, dentre as menos citadas estão ballet, jazz, vôlei, corrida, capoeira, queimada e jump. Vê-se que dois dias por semana é o maior número de dias destacados de praticar a atividade física $48,8 \%$.

Mediante a realização das medidas antropométricas e, por conseguinte o cálculo do Índice de Massa Corporal (IMC) nota-se que a variável eutrofia se sobressaiu em ambos os sexos. Vê-se que no gênero feminino do total das 64 alunas participantes $47(73,4 \%)$ das mesmas apresentam um peso ideal para a sua idade, sendo seguidas por 11 $(17,2 \%)$ com sobrepeso. Do total dos 55 estudantes do sexo masculino 43 $(78,2 \%)$ se encontram no peso adequado para a idade (TABELA 5).

Em relação à média da $\mathrm{PA}$ dos alunos participantes observa-se que prevalecentemente a PA normal. No sexo masculino, todos os $55(100 \%)$ estudantes integrantes da pesquisa apresentaram a média da sua pressão dentro dos limites normais. Dentre as 64 meninas $60(93,7 \%)$ das mesmas manifestaram a média da PA dentro dos limites normais, e as demais se dispuseram em limítrofe $2(3,1 \%)$, hipertensão estágio I 2 (3,2\%), conforme (TABELA 5).

\section{DISCUSSÃO}

O presente estudo objetivou identificar a prevalência da hipertensão arterial sistêmica bem como a predisposição dos escolares desenvolverem a mesma, através da associação dos fatores de risco. Os casos de hipertensão arterial na infância são importantes dizeres da saúde 
Obesidade (> Percentil 97 e $\leq$ Percentil 99,9)

\begin{tabular}{lcccc} 
Obesidade Grave (> Percentil 99,9) & - & - & - & - \\
\hline Total & 64 & 100 & 55 & 100 \\
\hline Média pressão arterial & $\mathbf{n ~ ( 6 4 )}$ & \multicolumn{4}{c}{$\mathbf{n ~ ( 5 5 )}$} \\
\hline Normal (< Percentil 90) & 60 & 93,7 & 55 & 100 \\
\hline Limítrofe (Entre Percentis 90 a 95) & 2 & 3,1 & - & - \\
\hline Hipertensão Estágio 1 (Percentil 90 a 95 mais 5mmHg) & 1 & 1,6 & - & - \\
\hline Hipertensão Estágio 2 (> Percentil 99 mais 5mmHg) & 1 & 1,6 & - & - \\
\hline Total & 64 & 100 & 55 & 100
\end{tabular}

Fonte: as autoras (2018)

cardiovascular no indivíduo adulto, já que crianças com valores pressóricos acima do percentil 90 frequentemente tornam-se adultos hipertensos, tornando assim os estudos de prevalência de hipertensão arterial em crianças e adolescentes importantes instrumentos de avaliação da saúde cardiovascular dessa população, tendo em vista o risco iminente de complicações cardiovasculares nesses indivíduos ${ }^{(8)}$.

Ao que concerne à pressão arterial o estudo demonstrou uma prevalência da PA normal em ambos os sexos, porém no sexo feminino houve uma pequena minoria com alteração na média das aferições. A hipertensão é mais comum no sexo feminino bem como fatores de risco nos quais podem desencadear a mesma, como no caso da obesidade devido aos hormônios, que se mostra mais frequente no gênero feminino ${ }^{(8)}$.

A raça prevalecente branca da amostra sugere relação com a não alteração da PA. Estudos recentes demonstram que em relação a raça/cor, os indivíduos negros alcançam as maiores médias de PA. Desta forma, as diferenças raciais e a HAS infantil são diretamente interligadas ${ }^{(9)}$.

Entre a maioria das crianças avaliadas a renda familiar gira entre um e até dois salários mínimos e a escolaridade dos pais ou responsável legal pelos mesmos variou entre ensino médio completo e fundamental incompleto. Ressalta-se que o baixo grau de escolaridade dos pais pode interferir diretamente na baixa renda familiar. A bibliografia apresenta a ligação entre a HAS e a baixa condição socioeconômica familiar pode levar a situações inadequadas de vida, nas quais predispõem ou podem estar integradas à maior prevalência de hipertensão arterial e de elementos de risco para elevação da mesma(10). Em contrapartida, outros mencionam que nos países em desenvolvimento, como o Brasil, famílias de melhor poder aquisitivo as crianças têm mais chances de apresentarem sobrepeso, bem como doenças cardiovasculares, dentre elas a HAS, quando comparadas às menos abastadas, e em regiões ou estados menos desenvolvidos, a proporção de obesos eleva-se conforme o aumento da renda ${ }^{(11)}$. Deste modo, o estudo evidenciou elevada prevalência de baixa escolaridade e baixa renda corroborando com os estudos descritos anteriormente, porém não identificamos a relação entre o baixo poder aquisitivo e o aumento da prevalência na elevação da PA.

O baixo peso ao nascer esta intimamente relacionada à prematuridade e é um preditor de doenças cardiometabólicas na fase infanto-juvenil e adulta. Recém-nascidos nascidos com o peso $<2.500 \mathrm{Kg}$, agem diferentes mecanismos de adequação a vida extrauterina, dentre os quais o crescente metabolismo dos carboidratos e a decorrente alta da adiposidade, nos quais aumentam o risco futuro de doenças crônicas, como hipertensão, conforme citam os estudos da teoria de Barker ${ }^{(12)}$. Contudo, no estudo realizado identificamos que as crianças integran- tes se assemelham a literatura, nasceram com o peso adequado e a termo, não sugerindo correlação entre a predisposição a HAS até mesmo sua alteração.

Quanto o tempo de amamentação superior a seis meses e os níveis pressóricos encontrados, Identificou-se que crianças que amamentaram no peito por um período superior a seis meses demonstraram menor prevalência de alterações na PA do que crianças que amamentaram por menos de seis meses. Dentre os vários benefícios dados pelo aleitamento materno, enfatiza a prevenção dos distintos fatores de riscos cardiovasculares, como obesidade, dislipidemias, diabetes e HAS. A implicação do aleitamento materno na prevenção HAS, está relacionada ao baixo teor de sódio do leite materno em relação aos alimentos industrializados, à presença de ácidos graxos insaturados de cadeia longa no leite materno e à possibilidade de prevenção do sobrepeso/obesidade ${ }^{(13,14)}$.

A presença de HAS e DM em pais ou avós ocorreram em grande parte da amostra estudada. A presença de tais patologias na família constitui um fator precipitador para desenvolver HAS, diabetes mellitus e outras doenças cardiovasculares ${ }^{(15)}$. A conexão entre a presença da enfermidade na família e a elevação dos níveis pressóricos em crianças. Porém no presente estudo não foi identificado aumento na prevalência de PA elevada nos discentes e a presença da patologia nos familiares ${ }^{(5)}$.

Apesar das DCNT aparecerem mais repetidamente na fase adulta, o reconhecimento de condutas prejudiciais à saúde tem sido demonstrado na literatura, com foco em indivíduos jovens. "Hábitos alimentares inadequados, níveis insuficientes de atividade física, obesidade e tempo excessivo em atividades de lazer tecnológico sedentárias são alguns dos comportamentos mais comuns durante a juventude"(15).

Quanto à ingestão habitual de frutas dos estudantes foi destacado o consu- 
mo de apenas uma fruta ao dia, sendo necessário o estimulo a um maior consumo ao dia. A ingestão diária de, pelo menos, 400 gramas de frutas, legumes e verduras, o correspondente a cinco porções ${ }^{(15)}$. O estímulo ao consumo desses alimentos diariamente pode prevenir o aparecimento de DCV. Ainda citam que esses alimentos são capazes de diminuir o processo inflamatório vascular, regulando os níveis pressóricos ${ }^{(5)}$.

Nessa pesquisa, o tempo de lazer tecnológico não influenciou na prevalência da obesidade e da PA elevada. A literatura elucida que a tecnologia vem apoderando-se cada vez mais na vida da maioria das crianças. Tal fato afasta os jovens das atividades físicas, deixando-os cada vez mais predispostos a desenvolver obesidade e seus fatores associados, além de triplicar os riscos de desenvolveram doenças cardiovasculares. Con- tudo se cita a família sendo a primeira fonte de influência é responsável por incutir ao longo do processo de educação e desenvolvimento, valores sobre os hábitos de vida saudáveis ou fatores como sedentarismo, má alimentação e ingestão excessiva de alimentos ${ }^{(14,15)}$.

Assim, salienta-se o diagnóstico de fatores de risco na faixa etária infanto-juvenil, a fim de prevenir complicações futuras e possibilitando a reversão do quadro.

\section{CONCLUSÃO}

A partir da realização deste estudo, identificamos a necessidade de refletir sobre os fatores de risco para o desenvolvimento da hipertensão e ampliar nossos olhares em relação á carência de políticas públicas efetivas para prevenção e tratamento dessa doença. É necessário que se promovam alterações no comportamento populacional, com enfoque em programas educativos, alimentação saudável, comunicação social, prática de exercícios físicos, bem como educação da mídia associadas a maus hábitos de saúde. Tais ações para serem mais bem aceitas pela população devem considerar a fase de desenvolvimento em que a criança se encontra o funcionamento familiar e as questões étnicas e de fundo cultural.

Nossos achados apontam a necessidade de maiores esforços do poder público no sentido de estabelecer políticas públicas voltadas para a nutrição, com o intuito de sensibilizar e conscientizar a família, a escola e a sociedade em geral para a importância de se evitarem atividades sedentárias, estimulando a prática de exercícios físicos e de alimentação saudável.

\section{Referências}

1. Rodrigues VC, Correia DMS, Santoro DC. A Hipertensão Arterial como principal fator de risco para insuficiência cardíaca: Revisão integrativa de literatura. Revista Nursing, 2019; 22(259):3465-3474. Disponivel em: http://www.revistanursing.com.br/revistas/259/pg107.pdf. Acesso em 10 Jul. 2020.

2. Nascimento LR, Monteiro LN, Pereira TSS, Mill JG, Molina MDCB. Hipertensão arterial em escolares de 7 a 10 anos: um estudo de casos persistentes de alteração de pressão arterial em Santa Maria de Jetibá/ES. Revista Brasileira Pesquisa Saúde. 2015; 17(4):76-84. Disponivel em: https://pdfs.semanticscholar.org/84c7/ a6f828b49321 ecdd1 be077d58efb0387108f.pdf. Acesso em 10 Jul. 2019.

3. Sociedade Brasileira de Cardiologia. $7^{\mathrm{a}}$ Diretriz Brasileira de Hipertensão Arterial. Arquivo Brasileiro de Cardiologia. 2016; 107(3):1-104, 2016. Disponivel em: https://www.scielo.br/pdf/abc/v107n3s3/pt_0066-782X-abc-107-03-s3-0007. pdf. Acesso em 10 Jul. 2019.

4. Cordeiro JP, Dalmaso SB, Anceschi AS, Sá FGS, Ferreira LG, Cunha MRH, Leopoldo AS, Leopoldo APL. Hipertensão em estudantes da rede pública de Vitória/ ES: influencia do sobrepeso e obesidade. Revista Brasileira de Medicina no Esporte, v. 22, n. 1, p.1-7, 2016. Disponivel em: https://www.scielo.br/pdf/rbme/ v22n1/1517-8692-rbme-22-01-00059.pdf. Acesso em 10 Jul. 2019.

5. Crispim PAA, Peixoto MRG, Jardim PCBV. Fatores de risco associados aos níveis pressóricos elevados em crianças de dois a cinco anos. Arquivo Brasileiro de Cardiologia. 2014; 102(1):39-46. Disponivel em: https://www.scielo.br/pdf/ abc/2013nahead/aop_5652.pdf. Acesso em 10 Jan. 2020.

6. Instituto Brasileiro de Geografia e Estatística. Estimativas populacionais para os municípios e para as Unidades da Federação brasileiros. Disponível em: $<$ https://www.ibge.gov.br/>. Acesso em: 24 Set. 2020.

7. Brasil, Ministério da Saúde. Orientações para coleta e análise de dados antropométricos em serviços de saúde. Norma técnica do Sistema de Vigilância Alimentar e Nutricional (SISVAN), $1^{\text {a }}$ ed., p. 1-72, 2011.

8. Ferreira JS, Aydos RD. Prevalência de hipertensão arterial em crianças e adolescentes obesos. Revista Ciência e Saúde Coletiva. 2010; 15(1):97-104, 2010. Disponível em: https://www.scielo.br/scielo.php?pi$\mathrm{d}=\mathrm{S} 1413-81232010000100015 \&$ script=sci_abstract\&tlng=pt. Acesso em: 20
Mar. 2019.

9. Souza CB, Dourado CS, Mill JG, Salaroli LB, Molina MDCB. Prevalência de hipertensão em crianças de escolas públicas. Revista International Journal of Cardiovascular Sciences. 2017; 30(1):42-51. Disponivel em: https://www.scielo. br/scielo.php?pid=S1413-81232010000100015\&script=sci_abstract\&tlng=pt. Acesso em: 20 Mar. 2019.

10. Silva SSBE, Oliveira SFSB, Pierin AMG. 0 controle da hipertensão em muIheres e homens: uma análise comparativa. Revista da Escola de Enfermagem da USP/SP. 2015; 50(1):50-58. Disponivel em: https://www.scielo.br/pdf/reeusp/ v50n1/pt_0080-6234-reeusp-50-01-0050.pdf. Acesso em: 20 Abr. 2019.

11. Souza MCC, Tibúrcio JD, Bicalho JMF, Rennó HMS, Dutra JS, Campos LG, Silva ES. Fatores associados à obesidade e sobrepeso em escolares. Revista Texto Contexto Enfermagem. 2014; 23(3):712-719. Disponível em: https://www.scielo. br/pdf/tce/v23n3/pt_0104-0707-tce-23-03-00712.pdf.Acesso em: 20 Mar. 2019. 12. Ribeiro AM, Lima MC, Lira PIC, Silva GAP. Baixo peso ao nascer e obesidade: associação causal ou casual? Revista Paulista de Pediatria. 2015; 33(3):340348. Disponivel em: https://www.scielo.br/scielo.php?script=sci_arttext\&pi$\mathrm{d}=$ S0103-05822015000300340. Acesso em: 20 Mar. 2020.

13. Ferreira SD, Carballo FP, Sousa FF, Silva DMR. Prevalência e fatores associados ao sobrepeso/obesidade e à hipertensão arterial sistêmica em crianças da rede privada de ensino de Divinópolis/MG. Caderno de Saúde Coletiva. 2015; 23(3):289-297. Disponível em: https://www.scielo.br/pdf/cadsc/v23n3/ 1414-462X-cadsc-23-3-289.pdf. Acesso em: 20 Mar. 2020.

14. Duré ML, Schwanke NL, Borges TS, BURGOS MS, Garcia EL, Krug, SF. A obesidade infantil: um olhar sobre o contexto familiar, escolar e da mídia. Revista de Epidemiologia e Controle de Infecção. 2015; 5(4):191-196. Disponivel em: https://online.unisc.br/seer/index.php/epidemiologia/article/view/6072/4635. Acesso em: 05 Jun. 2020.

15. Silva FMA, Menezes AS, Duarte MFS. Consumo de frutas e vegetais associado a outros comportamentos de risco em adolescentes no nordeste do Brasil. Revista Paulista de Pediatria. 2016; 34(3):309-315. Disponível em: https://www.scielo.br/ pdf/rpp/v34n3/pt_0103-0582-rpp-34-03-0309.pdf. Acesso em: 10 Jul. 2020. 